\title{
Spinal epidural hematoma after epidural anesthesia in a patient receiving enoxaparin -A case report-
}

\author{
In Soo Han, Eun Yong Chung, and Yun-Jin Hahn \\ Department of Anesthesiology and Pain Medicine, The Catholic University of Korea College of Medicine, Seoul, Korea
}

Spinal epidural hematoma is a rare but serious neurological complication of neuraxial anesthesia. Enoxaparin sodium is a low molecular weight heparin (LMWH) for use in preventing deep venous thrombosis in patients undergoing total hip arthroplasty and total knee arthroplasty. Hemorrhage is an uncommon but documented adverse reaction when using LMWH. We report a case of epidural hematoma after lumbar epidural anesthesia in a patient who administered enoxaparin in perioperative period. (Korean J Anesthesiol 2010; 59: 119-122)

Key Words: Enoxaparin, Epidural anesthesia, Spinal epidural hematoma.

Anticoagulants, antiplatelet drugs, glycoprotein IIb/IIIa antagonists, and thrombolytics are used for the prevention and treatment of stroke, myocardial infarction, deep vein thrombosis, and pulmonary embolism in many patients undergoing surgery [1]. Among them, low molecular weight heparin (LMWH) has been excellent safety and efficacy without need of laboratory monitoring. However, reports of spinal hematoma occurring spontaneously and in association with regional anesthesia [2-4] have generated concern about the safety of spinal or epidural anesthesia in patients receiving LMWH. We report a case of an epidural hematoma in a patient who was administered enoxaparin (LMWH) prophylactically against the development of deep vein thrombosis perioperatively and received lumbar continuous epidural anesthesia.

\section{Case Report}

An American Society of Anesthesiologists physical state III, 82-year-old man who had previously undergone both total hip arthroplasty was admitted for an elective left revisional total hip arthroplasty. He had bronchial asthma, moderate chronic airway limitation, and poor exercise tolerance. Pulmonary function test showed a forced expiratory volume in 1 second of $0.73 \mathrm{~L}$ (52\% predicted) and forced vital capacity of $2.09 \mathrm{~L}$ ( $94 \%$ predicted). He was treated with xanthines, mucolytics, and inhalater for bronchial asthma. Spinal radiograph showed moderate osteoporotic compression fracture of the lumbar and lower thoracic spine. His routine preoperative blood investigations revealed normal electrolytes, hematocrit

Received: September 21, 2009. Revised: October 5, 2009. Accepted: December 17, 2009.

Corresponding author: Eun Yong Chung, M.D., Department of Anesthesiology and Pain Medicine, The Catholic University of Korea College of Medicine, Bucheon St. Mary's Hospital, 2 Sosa-dong, Wonmi-gu, Bucheon 420-717, Korea. Tel: 82-32-340-2158, Fax: 82-32-340-2255, E-mail: anes36@catholic.ac.kr

(c) This is an open-access article distributed under the terms of the Creative Commons Attribution Non-Commercial License (http:// creativecommons.org/licenses/by-nc/3.0/), which permits unrestricted non-commercial use, distribution, and reproduction in any medium, provided the original work is properly cited. 
(Hct) of $35.6 \%$, platelet count of $440,000 / \mathrm{mm}^{3}$, prothrombin time of $90.5 \%$ (normal range, $72-161 \%$ ), activated partial thromboplastin time of 35.2 seconds (normal range, 21-38 seconds), with an international normalized ratio (INR) of 1.05 (therapeutic level, 0.7-1.24).

Perioperative thromboprophylaxis with enoxaparin (Aventis Pharma, Seoul, Korea) 40 mg subcutaneously once daily was planned by surgical team and initiated enoxaparin $20 \mathrm{mg}$ the evening before surgery. The next morning at 8 a.m, epidural anesthesia was performed by an experienced anesthesiologist. A 17-gauge Tuohy needle was introduced at the level of the L4-5 interspace using a midline approach and a 19-gauge epidural catheter (ARROW ${ }^{\circledR}$, Arrow International Inc., Reading, PA, USA) advanced $3 \mathrm{~cm}$ into the epidural space. The epidural space was identified using the loss of resistance technique and an air-filled syringe. The procedures were atraumatic with no paresthesia or blood in the catheter. The epidural test dose ( $3 \mathrm{ml}$ of $1.5 \%$ lidocaine with epinephrine $1: 200,000)$ and an additional 12 $\mathrm{ml}$ of $1.5 \%$ lidocaine was administered via epidural catheter. General anesthesia was conducted using a laryngeal mask airway (LMA) and maintained under sevoflurane-nitrous oxide-oxygen. Surgery was completed uneventfully and the catheter was removed intact before transporting him to the postanesthesia care unit. No blood was observed on the catheter or at the insertion site. Since we were anxious about the aseptic maintenance and the safety of using enoxaparin in a patient with indwelling catheter for postoperative analgesia, the catheter was removed in the operating room.

The patient recovered uneventfully from the epidural anesthetic. That evening, enoxaparin $40 \mathrm{mg}$ administered

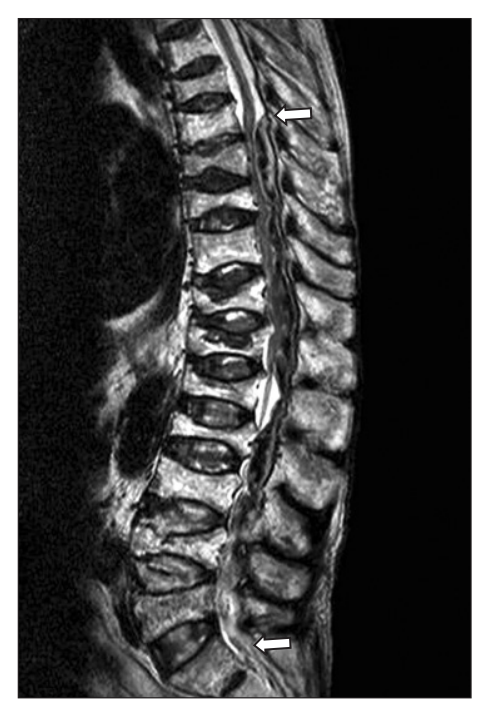

Fig. 1. Spine sagital magnetic resonance image showing the epidural hematoma (arrows) in the posterior epidural space along T8-L5 with resultant compression of the spinal cord and thecal sac. subcutaneously. The second dose time of the enoxaparin (LMWH) was 2 hours after the removal of the epidural catheter. On the evening of the second postoperative day, 4 hours after the fourth enoxaparin administration, the patient complained reduced sensation of the right, nonoperated leg as well as motor weakness of the left leg. No back pain was reported. Emergency magnetic resonance imaging revealed T8-L5 epidural hematoma with cord compression (Fig. 1). Emergency decompressive laminectomy to evacuate the hematoma was performed. There was a delay of 5 hours after the neurosurgical decision because of family's refusal. No vascular malformation was seen. Before the second surgical procedure, coagulation profile was showed as hemoglobin $6.4 \mathrm{~g} / \mathrm{dl}$, Hct $18.9 \%$, prothrombin time $49.8 \%$, activated partial thromboplastin time 63.1 seconds, international normalized ratio 1.49 , fibrinogen 71 $\mathrm{mg} / \mathrm{dl}$ (normal range, 80-415), antithrombin III 54\% (normal range, 60-90\%), D-dimer $461 \mathrm{ng} / \mathrm{ml}$ (normal range, 0-322), and a platelet count of $129,000 / \mathrm{mm}^{3}$. Postoperatively there was no improvement in neurologic function. Twelve packed red blood cells and 5 fresh frozen plasmas were used from the initial operation to the second operation. The patient subsequently expired from myocardial infarction attack 2 days later.

\section{Discussion}

Patients undergoing total hip arthroplasty are at increased risk for deep venous thrombosis and pulmonary embolism. Without prophylaxis, the prevalence of deep venous thrombosis is $51 \%$ [5] and that of fatal pulmonary embolism is $2 \%$ [6]. The fact that regional anesthesia reduces the risk of venous thromboembolism is well known. LMWH is an effective antithrombotic agent for total hip arthroplasty patients. Therefore, neuraxial anesthesia and postoperative analgesia with early pharmacologic prophylaxis would appear to be the most efficacious approach toward preventing thromboembolic complications.

LMWH has become a popular drug in many institutions because it provides safe and effective prophylaxis of deep venous thrombosis. However, its use is being associate with an increasing incidence of spinal hematoma following neuraxial blockade. Estimates of spinal hematoma in patients receiving LMWH were higher at $33: 100,000$ in those undergoing epidural technique and $1: 100,000$ in those receiving spinal anesthesia [7] compared with incidence of 1 : 150,000 after an epidural block and $1: 220,000$ after a spinal block [8]. Anatomical, technical, and pharmacological factors, including vertebral spine abnormalities, traumatic spinal tap, multiple attempts at needle placement, and use of antiplatelet and anticoagulant therapy may be implicated in formation of spinal epidural hematoma [4]. Several factors suspected of predisposing patients to 
epidural hematoma include a dose of enoxaparin that exceeds the recommended dose, administration of enoxaparin to surgical patients before the establishment of hemostasis, use of epidural catheters, concomitant administration of medications affecting hemostasis, presence of vertebral column abnormalities, advanced age, and female gender [3,4,9]. The study performed by Vandermeulen et al. [9] revealed that major of spinal hematoma (87\%) resulted from impaired hemostais or difficulty in needle placement. In the country, there is a epidural hematoma case after epidural catheterization without coagulopathy [10]. The etiology of spinal epidural hematoma is unknown, but Miyazaki et al. [11] mentioned that a small injury or high intracavity pressure could lead to rupture and hematoma since posterior vertebral and epidural veins are very fragile.

LMWH have a dose-dependent bleeding potential [12,13]. It is interesting to note that in North America the approved dose of enoxaparin is $30 \mathrm{mg}$ twice daily while in Europe a 40 mg single daily dose is used $[4,5,9,14]$. The higher dose used in North America may have contributed to the apparent increase in anticoagulant related epidural hematoma [9,12-14]. This case used European practice guidelines for the use of LMWH. The use of low dose LMWH does not usually require monitoring of the anticoagulant effect because of the predictability of its effect $[12,13]$. However, the use of high dose LMWH in elderly patients with multisystem disease probably requires laboratory monitoring [12]. It would be likely that in the elderly, there is an impairment of the renal function with decreased drug clearance, although there are many factors that alter drug levels and sensitivity in old people. The activated partial thromboplastin time and anti-Xa level are measures of LMWH anticoagulant effect. Peak anti-Xa levels of $0.1-0.2 \mathrm{U} / \mathrm{ml}$ provide safe and effective venous thromboembolism prophylaxis after hip or knee replacement surgery [14]. The timing of the dose of LMWH has a noctable effect on the occurrence of major bleeding. LMWH rapidly achieves peak levels within 3 to 5 hours after subcutaneous administration and reached $50 \%$ of their peak values 12 hours after injection $[4,9]$. The timing of the initial dose in orthopedic patients varies, with LMWH administered 12 hours preoperatively in Europe and 12-24 hours postoperatively in North America [4,14]. European practice guidelines may in fact be a good approach for intraoperative DVT. But, because hemostasis is not established in most patients until twelve to twenty four hours postoperatively, delaying the administration of the first dose of enoxaparin until this point ensures that the wound has stabilized. The acute spinal epidural hematoma in this elderly patient may be caused by spontaneous bleeding in the presence of relatively high dose enoxaparin through administration of enoxaparin before the establishment of hemostasis.
Catheter removal is also another important consideration in epidural anesthesia. Vandermeulen et al. [9] reported that spinal anesthesia was less traumatic than epidural anesthesia and catheter removal was significant in the development of spinal bleeding. Removal of catheter appeared to more critical than the insertion of epidural catheter in the genesis of a spinal hematoma, because bleeding occurred at removal moment in almost half of the cases [9]. Several authors [9,14] suggested that catheter removal and the patient's coagulation status at the time of removal are critical factors in the development of spinal bleeding. Miyazaki et al. [11] reported that in 50\%, the initial symptoms of spinal epidural hematoma were recognized within 24 hours of the removal of an epidural catheter and the injury to vessels caused by removal of an epidural catheter can lead to formation of spinal epidural hematoma. The major of cases occurred with catheter removal, suggesting that the catheter provided a mechanical tamponade that becomes dislodged with catheter removal. We deduce epidural hematoma from impaired hemostasis due to continuative enoxaparin therapy in the postoperative period. The American Society of Regional Anesthesia (ASRA) recommended that one must wait at least 10 to 12 hours after the last dose of LMWH to withdraw the catheter and then wait another 2 hours before giving the next LMWH dose $[1,8,9,13]$. In this case, the epidural catheter was removed 16 hours after the first dose of LMWH and the second dose of LMWH was administered 2 hours after catheter removal. Despite adhering to ASRA guideline concerning LMWH application, and despite the fact that preoperative clotting tests were normal, this patient developed an epidural hematoma 2 days after removal of epidural catheter. Accordingly, even if an atraumatic epidural or spinal anesthesia is, special precautions must be observed when removing the catheter in the patient administering LMWH.

It is very important to diagnose spinal hematomas early on so that prompt investigation and treatment are initiated. The first neuromuscular symptom of spinal hematoma was muscle weakness in $46 \%$ of patients and sensory deficit in $14 \%$ of patients in the work performed by Vandermeulen et al. [9]. Although the cardinal symptom of epidural hematoma is severe back pain, most patients reported sensory or motor deficits that progressed to paralysis, with the onset of symptoms occurring about 2 to 3 days after enoxaparin therapy had been initiated $[3,4]$. So, monitoring for signs of cord compression in the perioperative period is crucial with the typical initial complaint being new-onset weakness or numbness, not severe radicular back pain, as one might suspect. This patient also had not severe back pain but progressive numbness and weakness. Clinical factors influencing the neurologic prognosis are the severity of the pre-operative neurologic deficits, speed of onset of clinical signs, location of hematoma, age, duration of spinal 
cord compression, and the operative interval between onset of clinical signs and surgical evacuation $[9,11,15]$. It may also be possible that increased vigilance and early surgical intervention herald better outcomes. Although we followed the lead of ASRA guidelines about the LMWH application, the abnormal neurology showed 3 days after initiation of LMWH therapy and 2 days after catheter removal. We consider five points to be of possibility in this case. First, impaired renal function with decreased drug clearance in the elderly may result in drug levels and sensitivity changes. Second, even if the procedure itself was atraumatic, the injury to vessels caused by removal of epidural catheter could form spinal epidural hematoma. Third, the next LMWH within relatively short time (2 hours) of catheter removal might aggravate the patient's hemostasis for 12 to 24 hours postoperative period. Fourth, the consecutive LMWH administration without adjustment of coagulation state might cause the enlargement of hematoma. Finally, the disseminated intravascular coagulation through massive transfusion also may break down the patient's homeostasis, and result in the expansion of hematoma volumes. Response to subcutaneous LMWH therapy remains speculative, as subcutaneous blood flow may vary considerably interindividually in the elderly and may result in therapeutic anticoagulation. So, since the elderly is a risk factor for bleeding during treatment with $\mathrm{LMWH}$, careful evaluation of individual hemorrhagic risk, a slight decrease of doses, atraumatic technique and careful laboratory monitoring are recommended.

In conclusion, the potential development of a spinal hematoma in patients receiving neuraxial anesthesia must always be kept in mind whether of not an anticoagulant was used. Patients anticoagulated or scheduled to be anticoagulated with LMWH or heparinoids for prevention of thromboembolic complication are at risk of developing an epidural or spinal hematoma after neuraxial anesthesia or spinal puncture. The risk of these events is increased by the use of indwelling epidural catheters for administration of analgesia or by the concomitant use of drug affecting hemostasis such as non steroidal anti-inflammatory drugs, platelet inhibitors, or other anticoagulants or by old age. Since early diagnosis of an epidural hematoma after initial symptom and early surgery might have improved outcome, we should closely monitor patients for early signs of cord compression, such as progressive numbness or weakness, and bowel and bladder dysfunction after lumbar epidural anesthesia in a patient who administered enoxaparin in perioperative period.

\section{References}

1. Donegan E, Stratmann G, Kan TN. Hemostasis. In: Basics of Anesthesia. 5th ed. Edited by Stoelting RK, Miller RD: Philadelphia, Churchill Livingstone. 2007, pp 331-46.

2. Groen RJ, Ponssen H. The spontaneous spinal epidural hematoma. A study of the etiology. J Neurol Sci 1990; 98: 121-38.

3. Wysowski DK, Talarico L, Bacsanyi J, Botstein P. Spinal and epidural hematoma and low-molecular-weight heparin. N Engl J Med 1998; 338: $1774-5$

4. Chan L, Bailin MT. Spinal epidural hematoma following central neuraxial blockade and subcutaneous enoxaparin: a case report. J Clin Anesth 2004; 16: 382-5.

5. Clagett GP, Anderson FA Jr, Heit J, Levine MN, Wheeler HB. Prevention of venous thromboembolism. Chest 1995; 108(Suppl 4): 312S-34S.

6. Haake DA, Berkman SA. Venous thromboembolic disease after hip surgery. Risk factors, prophylaxis and diagnosis. Clin Orthop Relat Res 1989; 242: 212-31.

7. Schroeder DR. Statistics: detecting a rare adverse drug reaction using spontaneous reports. Reg Anesth Pain Med 1998; 23: 183-9.

8. Tryba M. Epidural regional anesthesia and low molecular heparin: pro. Anasthesiol Intensivmed Notfallmed Schmerzther 1993; 28: 179-81.

9. Vandermeulen EP, Van Aken H, Vermylen J. Anticoagulants and spinal-epidural anesthesia. Anesth Analg 1994; 79: 1165-77.

10. Choe WJ, Choe JH, Kim JW. Epidural hematoma after epidural catheterization without coagulopathy: a case report. Korean J Anesthesiol 2006; 50: 221-3.

11. Miyazaki M, Takasita M, Matsumoto H, Sonoda H, Tsumura H, Torisu T. Spinal epidural hematoma after removal of an epidural catheter: case report and review of the literature. J Spinal Disord Tech 2005; 18: 547-51.

12. Yin B, Barratt SM, Power I, Percy J. Epidural haematoma after removal of an epidural catheter in a patient receiving high-dose enoxaparin. Br J Anaesth 1999; 82: 288-90.

13. Hynson JM, Katz JA, Bueff HU. Epidural hematoma associated with enoxaparin. Anesth Analg 1996; 82: 1072-5.

14. Horlocker TT, Heit JA. Low molecular weight heparin: biochemistry, pharmacology, perioperative prophylaxis regimens, and guidelines for regional anesthetic management. Anesth Analg 1997; 85: 874 85.

15. Lawton MT, Porter RW, Heiserman JE, Jacobowitz R, Sonntag VK, Dickman CA. Surgical management of spinal epidural hematoma: relationship between surgical timing and neurological outcome. J Neurosurg 1995; 83: 1-7. 